\title{
RESIGNIFICAR LAS PRÁCTICAS EDUCATIVAS URUGUAYAS: COMPROMISO DE TODOS. RED DE DOCENTES NOVELES DE FÍSICA EN PAYSANDÚ
}

\author{
Redefining Uruguayan educational practices is everyone's \\ commitment. Network of novice physics teachers in Paysandú
}

\author{
Marie Noel Vallo \\ Consejo de Formación en Educación, ANEP \\ Departamento de Física \\ IFD de Paysandú, Uruguay \\ mvallo@docente.ceibal.edu.uy

\section{Ma. Giselle Vargas} \\ Consejo de Formación en Educación, ANEP \\ Departamento de Física \\ IFD de Paysandú, Uruguay \\ magisevargas@gmail.com

\section{Virginia Melo} \\ Consejo de Formación en Educación, ANEP \\ Departamento de Física \\ IFD de Paysandú, Uruguay \\ melovirginia0@gmail.com
}

Recibido: 13 de setiembre de 2020

Aceptado: 22 de diciembre de 2020

\section{Resumen}

El siguiente trabajo se basa en los resultados de la investigación-acción realizada en el período 20172018 sobre una red de acompañamiento a docentes noveles de física en la ciudad de Paysandú (Uruguay), en el marco de la convocatoria del Fondo Sectorial de Educación ANII-CFE Investiga 2016. La red de acompañamiento fue creada con miras a facilitar la inserción profesional de los docentes egresados y consolidar experiencias innovadoras, buscando la resignificación de las prácticas áulicas. Adecuar la enseñanza de la Física al estudiante del siglo XXI implica un cambio necesario que podría concretarse de la mano de los docentes noveles que aporten "aire fresco" al sistema, siempre y cuando se sientan apoyados y continúen su formación fortaleciendo su profesionalización. Sin embargo, diversos antecedentes indican que el novel docente recurre a prácticas de su biografía escolar, manteniéndose en su zona de confort si no encuentra el apoyo necesario para innovar. El programa de acompañamiento llevado a cabo en esta investigación se presentó como una oportunidad de concretar ese apoyo y conformar redes interpersonales que lograron mantenerse en el tiempo hasta el presente, facilitando a los docentes noveles desarrollar el trabajo colaborativo y afianzar sus competencias para una buena enseñanza.

Palabras claves: Docente novel, Redes de acompañamiento, Enseñanza de la física.

\footnotetext{
Abstract

The following work is based on the results of the action-research carried out in the period 20172018 on a support network for novice physics teachers. The research was developed within the framework of the call for the Sectorial Education Fund ANII-CFE Investiga 2016. The support network was created with the goal of facilitating the professional insertion of graduate teachers and
} 
consolidating innovative experiences, seeking the resignification of classroom practices. Adapting Physics teaching to the student of the XXI century implies a necessary change that could take place in the hands of novice teachers who provide "fresh air" to the system. But to successfully improve teaching practices with innovation, new teachers need to feel supported as much as continue their training, strengthening their professionalization. However, various antecedents indicate that the novice teacher resorts to practices from his school biography, staying in his comfort zone if he does not find the necessary support to innovate. The accompaniment program carried out in this research was presented as an opportunity to provide that necessary support and create interpersonal networks that have managed to maintain themselves in time up to the present facilitation new teachers to develop collaborative work and strengthen their skills for good teaching.

Keywords: Novice teachers, Support networks, Physics teaching

\section{Introducción}

En este documento se describe la investigación llevada a cabo entre los años 2017 y 2018 sobre una red de acompañamiento a docentes noveles de física creada a tales efectos; aportando información sobre el diseño e implementación de la red y sobre la evaluación de los resultados obtenidos en este primer acercamiento al tema. El término red de acompañamiento refiere a los diferentes vínculos establecidos, mediados en parte por la tecnología, entre docentes de física noveles e investigadoras - docentes de didáctica de la física- con el fin de mejorar las prácticas de aula, afianzar y compartir estrategias de enseñanza y aprendizaje basadas en la innovación y reflexión.

El estudio se desarrolló con base en el Instituto de Formación Docente de Paysandú, participando del mismo los docentes noveles de Física egresados de la misma Institución en los últimos cuatro años (anteriores a la investigación) y que se desempeñen como docentes en Educación Media.

Desde el rol investigador-formador, partimos de las siguientes interrogantes: ¿Cuáles son las necesidades del profesor novel para su inserción amigable en las instituciones educativas? ¿Influye la formación continua en las prácticas áulicas? ¿Qué respuestas y qué importancia le adjudicamos como colectivo docente a las interrogantes anteriores? En consecuencia, se diseña un programa de acompañamiento con características de coparticipación, con el objetivo principal de mejorar las prácticas de aula a través del afianzamiento de las relaciones entre docentes. La investigación, realizada en modalidad investigación-acción, busca respuestas sobre las ventajas o beneficios de este programa para la mejor inserción de los noveles y su mejor desempeño en el aula.

\section{Objetivo general:}

Indagar sobre la inserción profesional de los docentes noveles de Física, explorando si las redes de acompañamiento constituyen una metodología válida de andamiaje que les permita integrarse armoniosamente en el sistema educativo, desarrollando buenas prácticas innovadoras y reflexivas.

\section{Objetivos específicos:}

- Gestionar un programa de acompañamiento a docentes noveles a través de redes interpersonales, donde los participantes vayan adquiriendo protagonismo en la coparticipación 
- Evaluar el interés y compromiso de los docentes noveles frente a un programa de acompañamiento

- Detectar las dificultades con las que se enfrentan los docentes noveles en su inserción laboral

- Reflexionar sobre las prácticas pre profesionales y pos profesionales, cuestionando la armonía entre ambas y promoviendo la autorreflexión

- Indagar si un programa de acompañamiento permite que los docentes noveles desarrollen su potencial y produce mejoras en sus prácticas educativas

\section{Desarrollo}

\subsection{Marco teórico}

Los primeros años de trabajo es una etapa en la cual coexisten múltiples miradas en búsqueda de la voz de los propios noveles. Una investigación llevada a cabo por Maciel (2016) concluye en el reconocimiento de los profesores principiantes de una necesaria formación profesional continua, que les facilite herramientas para superarse $\mathrm{y}$, a la vez, de la necesidad del apoyo institucional que logre contener las necesidades de los noveles. Nossar (2016) señala que las principales preocupaciones de los docentes noveles se refieren tanto al "dominio conceptual" como al "control de la disciplina en el aula” (p.13). Maciel (2016) indica que los noveles manifiestan, entre otras, la necesidad de "saber tratar a los alumnos adolescentes y las conductas inadecuadas de los mismos, de motivarlos por el aprendizaje, de contribuir con la creación de la cultura del centro educativo desde la perspectiva relacional" (p.92). El fortalecimiento profesional de los noveles docente se desprende de las posibilidades de formación y de retroalimentación propiciado por el acompañamiento, con apoyo a la reflexión e investigación de sus propias prácticas atendiendo a mejorar la enseñanza de la física.

Davini (2015) considera que la formación de grado genera los cimientos que habilitan a ejercer la profesión, pero advierte que todo lo que se haga en ese período "es olvidado o abandonado durante el ejercicio de la docencia en la socialización laboral una vez graduados” (p. 22). El novel docente, sin apoyos institucionales o de algún colega innovador, tiende a reproducir rutinas tradicionales que forman parte de su biografía escolar y que requieren menor compromiso. "La distancia entre la teoría que reciben en su formación inicial y la realidad con la que se encuentran en los centros educativos" (Nossar, 2016, p.102) donde deben ponerla en práctica desorienta al docente principiante, que no encuentra recursos suficientes en su formación para enfrentar su tarea. Klein (2012) considera que, para mejorar la inserción profesional de los docentes de física, uno de los aspectos necesarios es que la "institución formadora" apoye a dichos noveles "apoyando la exploración y puesta en funcionamiento de experiencias innovadoras, que logre una fundamentación sólida en su accionar y más "plástica" para captar nuevas propuestas y proponentes" (p.161).

En la planificación del programa de acompañamiento, se tiene en consideración que todo dispositivo de acompañamiento pedagógico es una construcción social y como tal responde a las necesidades sociales y es influenciado por diferentes aspectos sociales y culturales. A la vez son construcciones técnicas e incluyen concepciones pedagógicas. De acuerdo a Souto (1999), surgen como respuesta a problemas de la acción, son creados, pensados y llevados a cabo por personas, sujetos que los con- 
ciben y concretan seleccionando estrategias y marco teórico adecuado, gestionando lugares y tiempos para su ejecución. Para el diseño del dispositivo se parte, de los problemas que se enfrenta el grupo de noveles que se va a acompañar y de la información recabada en las visitas iniciales y entrevistas, para acercarnos a la complejidad de la situación. La siguiente tabla informativa (Tabla 1) muestra los aspectos que se tuvieron en cuenta en la planificación del programa de acompañamiento:

\section{Tabla 1: Principales ejes teóricos considerados en la planificación del programa (Elaboración propia)}

\begin{tabular}{|c|c|}
\hline $\begin{array}{c}\text { De la teoría a } \\
\text { la técnica }\end{array}$ & $\begin{array}{l}\text { Los dispositivos planificados están cruzados por nuestra subjetividad, y, a la } \\
\text { vez, deben responder a una realidad externa a quien los planifica. Ello conduce } \\
\text { a aceptar la plasticidad como parte de los mismos, e ir adecuando y moldeando } \\
\text { el proyecto de acompañamiento a la medida de y con los sujetos del mismo: los } \\
\text { docentes noveles. Barbier (1999) menciona la transformación del sujeto implícita } \\
\text { en la formación que, a la vez, contribuye a una transformación y reconstrucción } \\
\text { del espacio de acompañamiento a medida que se desarrolla en el tiempo. }\end{array}$ \\
\hline $\begin{array}{l}\text { "Shock de } \\
\text { la realidad" } \\
\text { (Veeman, } \\
\text { 1984) }\end{array}$ & $\begin{array}{l}\text { Es necesario diagnosticar en qué aspectos específicos se concreta ese "shock" } \\
\text { para el grupo objetivo, tener en cuenta las diferentes características de los siste- } \\
\text { mas educativos y niveles donde se insertan los noveles, señalando la importancia } \\
\text { de contextualizar los programas de acompañamiento. } \\
\text { Para complementar la contextualización este programa incluye en su etapa inicial } \\
\text { entrevistas con los equipos de dirección e inspección de las instituciones involu- } \\
\text { cradas, en el entendido de que cada dispositivo de acompañamiento se concreta } \\
\text { en un espacio y tiempo determinados. }\end{array}$ \\
\hline $\begin{array}{l}\text { Oportunidad } \\
\text { de reflexión }\end{array}$ & $\begin{array}{l}\text { El modelo en el que se enmarca este proyecto de acompañamiento se adhiere al } \\
\text { denominado "modelo hermenéutico reflexivo" (De Lella, 1999). Se entiende que } \\
\text { el docente es un profesional creativo y reflexivo en y de su práctica. Se concibe } \\
\text { la enseñanza como una tarea compleja que requiere opciones éticas y políticas de } \\
\text { las cuales no existen recetas ni experiencias previas. }\end{array}$ \\
\hline $\begin{array}{c}\text { Los ateneos o } \\
\text { talleres }\end{array}$ & $\begin{array}{l}\text { Estas modalidades de trabajo en grupo son priorizadas en la modalidad de acom- } \\
\text { pañamiento llevada a cabo. Se busca facilitar el aprendizaje y la formación. Según } \\
\text { Souto (1999) constituyen un "revelador" de lo subjetivo, un "analizador" de lo } \\
\text { revelado y un "provocador" de cambios. } \\
\text { A partir de ellas se busca sistematizar, volver a la acción y analizarla, cuestionarla } \\
\text { y abrirse a las posibilidades de cambios: "reflexividad" (Vera-Godoy, 2008). }\end{array}$ \\
\hline
\end{tabular}

\subsection{Metodología}

La estructura del programa de acompañamiento, enmarcada dentro del proyecto de investigación, consiste en una línea de trabajo donde se emplean diseños metodológicos cuantitativos y cualitativos, planificados y seleccionados para las diferentes etapas de su realización. Se consideró pertinente la aplicación de metodologías de investigación-acción en perspectiva empírico-analítica y humanístico-interpretativa que se describen a continuación.

Población objetivo: La población investigada estuvo integrada por los/las egresado/as del profesorado de Física del Instituto de Formación Docente de Paysandú entre 2014 y 2017 que se encontraran trabajando en Educación Media en el momento de la investigación. Al inicio de la investigación, setiembre de 2017, este grupo estaba compuesto por ocho docentes, que reunían las condiciones mencionadas. Se realizó 
una jornada de sensibilización donde se solicitaron voluntarios para participar de la investigación y en la cual todos se ofrecieron a ser elegidos para participar. El equipo de investigación había considerado que solamente podrían estudiarse cuatro casos, para realizar un seguimiento adecuado. En consecuencia se seleccionó una muestra de cuatro docentes dentro del grupo indicado. En la selección se tuvo en cuenta el grado de interés manifiesto en la jornada inicial junto a un criterio de conveniencia: horarios compatibles para realizar las visitas y entrevistas. Sin embargo, considerando que todos ellos habían manifestado su interés de participar, siempre fueron invitados en las instancias grupales, talleres y jornadas de formación. También se los incluyó en los grupos creados a efectos de la investigación, dejando de lado la evaluación de sus prácticas. Dado que la investigación no acompañó el año lectivo, en 2018 algunos egresados se unieron a la población inicial y una docente se trasladó a otro departamento, no afectando la muestra seleccionada.

El siguiente infograma (figura 1) presenta el calendario de actividades desarrolladas a lo largo de los 18 meses de implementación de la investigación.

\section{DESARROLLO DE LA INVESTIGACIÓN}

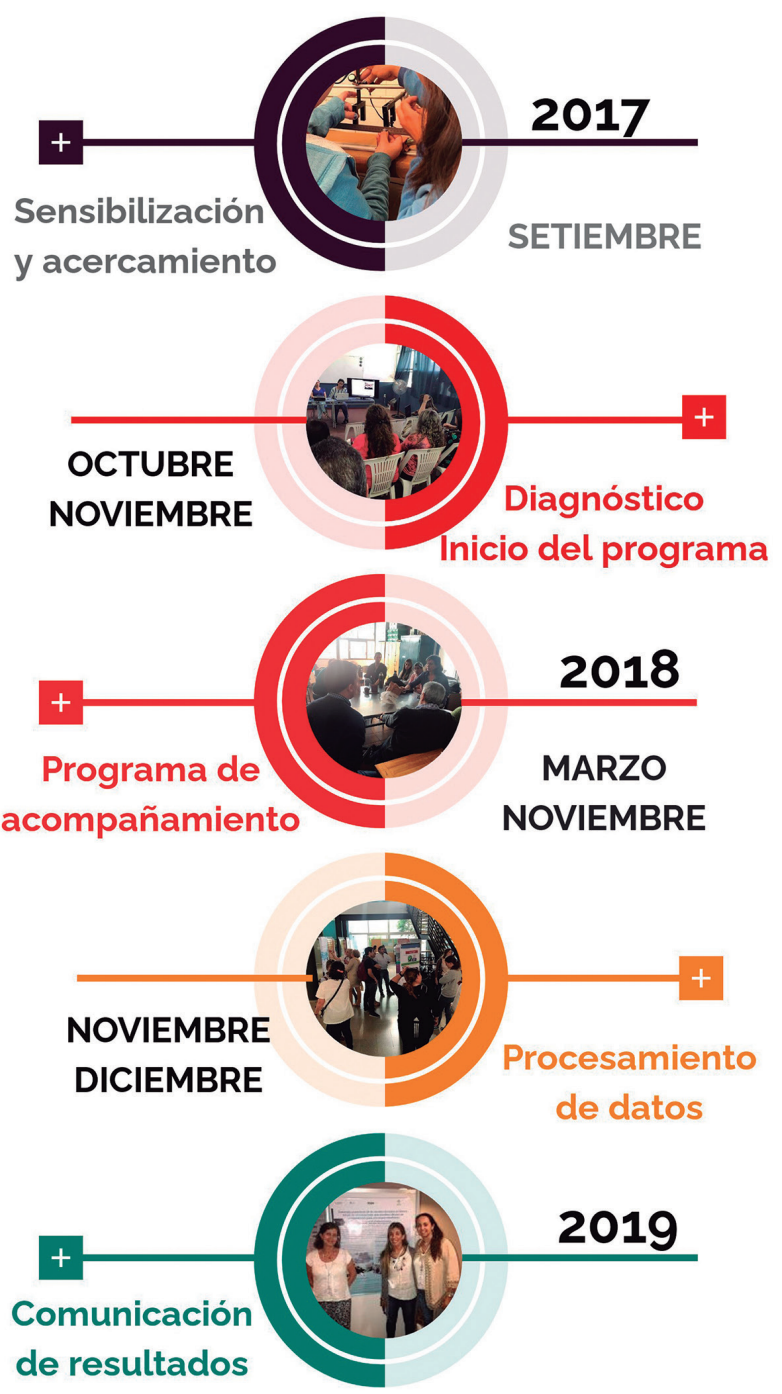

Figura 1: Calendario de actividades 
1) Sensibilización: Se organiza una reunión inicial explicativa con la totalidad de docentes noveles sobre el programa de acompañamiento y la investigación a realizar. Se realiza la selección de la muestra (cuatro docentes dentro de quienes se ofrecieron en forma voluntaria a participar).

2) Diagnóstico: Se realizan entrevistas y visitas a clases a los docentes noveles seleccionados. Se realizan encuestas y entrevistas a otros actores de las instituciones educativas: directores, ayudantes preparadores, inspectores de asignatura y de institutos y liceos, lo que permite tener un panorama desde diferentes miradas sobre la inserción de los docentes noveles y las dificultades que puedan percibirse.

3) Tejiendo redes: Se organiza y lleva a cabo el programa de acompañamiento, co-gestionado entre noveles e investigadoras. Se realizan encuentros presenciales que incluyen ateneos de discusión y espacios de formación, se concreta la creación de un grupo en la plataforma CREA2 y de uno de WhatsApp. Estas redes incluyen a la población completa, dado que los vínculos entre docentes noveles es parte del objeto a investigar.

\section{Desarrollo de la experiencia}

En el desarrollo de la experiencia del proyecto de acompañamiento se llevaron a cabo diferentes metodologías de trabajo:

- Instancias de formación: se organizaron talleres o jornadas con profesores/as visitantes expertos, idóneos en algunas áreas vinculadas a la enseñanza de la física, y otras dirigidas por el equipo de investigación y noveles voluntarios.

- Talleres de discusión o ateneos: Se organizaron encuentros presenciales entre los profesores noveles e investigadoras periódicamente, en los cuales se generaron instancias significativas de discusión y reflexión. Se compartió sobre actividades experimentales, metodologías de enseñanza y evaluación, recursos y TIC en el aula de física, entre otros. Avanzado el programa se fueron re definiendo instancias acordando con los intereses de los involucrados. En 2018, de común acuerdo con el grupo de noveles, el equipo de investigación resolvió invitar a participar a recientes noveles que iban egresando de la formación del profesorado de física, además de estudiantes avanzados que realizan la práctica docente con grupo a cargo y a docentes expertos.

- Trabajo en redes: Se crea un grupo en la plataforma ceibal CREA 2, gestionado por una docente novel. En este grupo se comparte material que sirve de insumo para las clases de todos los involucrados y, a la vez, se retroalimenta las instancias presenciales. El material compartido fue subido por distintos noveles y por las investigadoras: información, sitios web de los docentes participantes y otros, pruebas Kahoot, material bibliográfico, planificaciones, publicaciones, recursos web, canales de YouTube, videos y otros.

- Encuentros con estudiantes de la región: Se invitó a los docentes noveles a participar del encuentro de profesorados de la UBA, Argentina, con presentación de pósteres. Se organizó una actividad similar a nivel regional, con taller sobre pósteres, como cierre del acompañamiento (figura 2). 


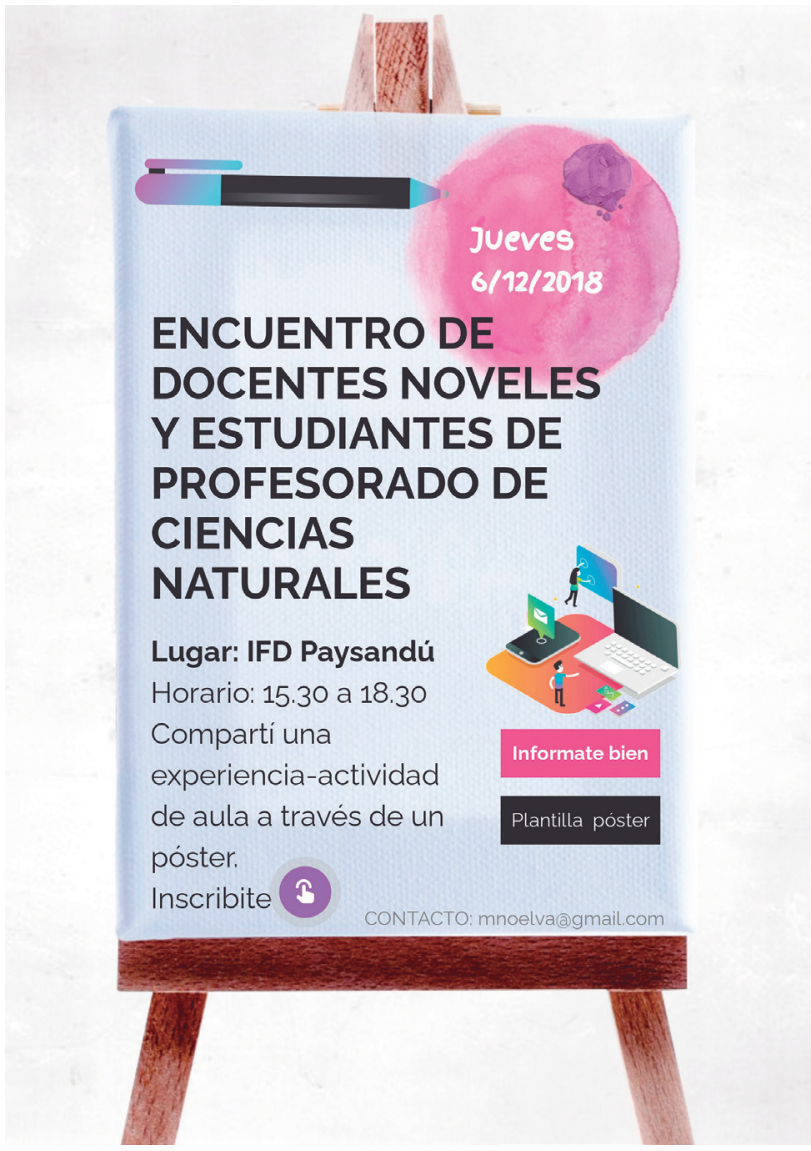

Figura 2: Invitación digital al encuentro. (Elaboración propia)

- Evaluación de impacto: Por un lado, se evalúa la participación en las redes (cantidad y calidad) y en los encuentros, en cuanto a asiduidad (participación de forma continuada) e interés. Se considera que la asistencia es un indicador del interés, pero también la calidad de la participación en los mismos, así como los aportes o cuestiones compartidos en las redes. Esto engloba la iniciativa para convocar encuentros de discusión y/o formación. Por otro lado, se recaba la opinión de los involucrados en el acompañamiento a través de entrevistas y encuestas finales. En el transcurso de la investigación se decidió evaluar la participación tanto en la muestra investigada como en el grupo completo de noveles.

\section{Análisis y discusión de datos}

Finalizado el programa de acompañamiento se analiza el grado de participación de los docentes noveles en las distintas actividades. El siguiente gráfico (figura 3) muestra la participación de los docentes investigados y del total de la población involucrada en las instancias de actividades presenciales a lo largo del programa de acompañamiento. Al no acompañar el año lectivo el total de noveles refiere a ocho (8) en 2017 y diez (10) en 2018. El gráfico muestra que las instancias presenciales fueron acompañadas en forma irregular por los docentes, muchas veces por problemas de agenda o personales (de acuerdo a los testimonios de los involucrados en el momento $\mathrm{y}$ en las entrevistas finales). 


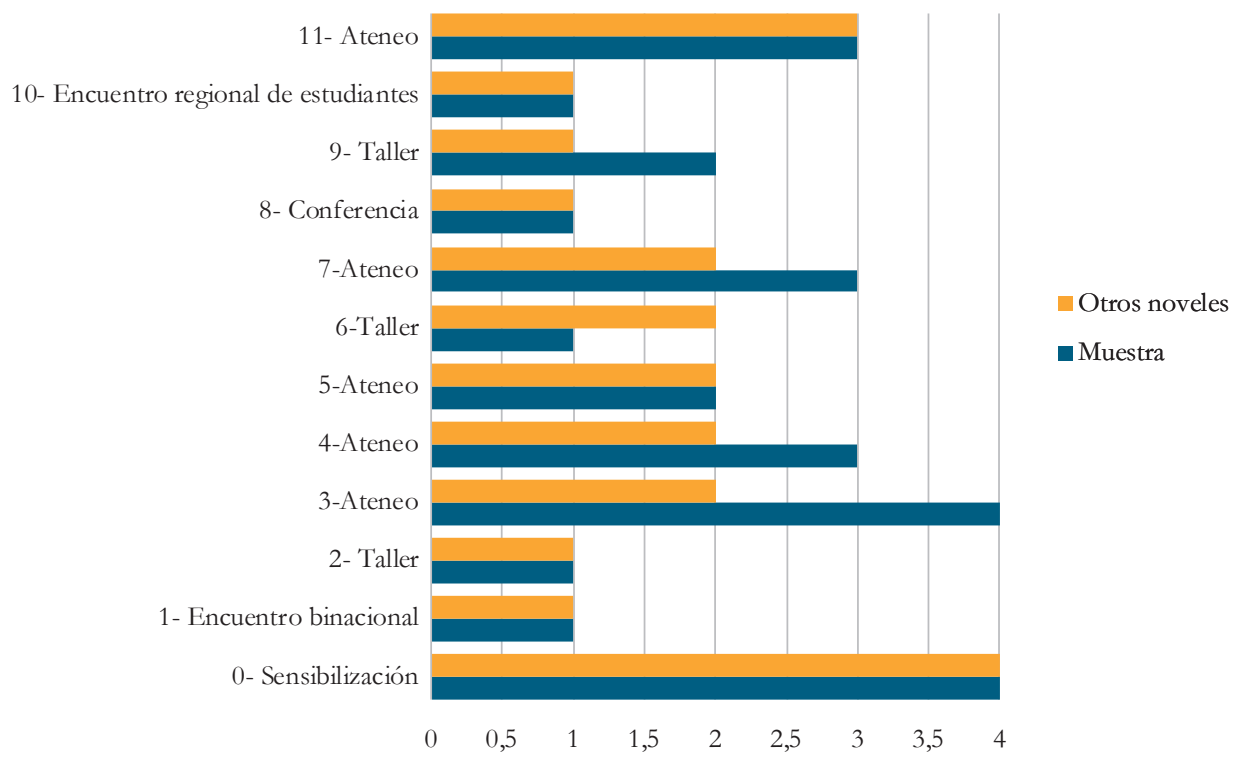

Figura 3: Participación de docentes noveles en cada instancia presencial. Elaboración propia en base a los datos obtenidos.

El siguiente gráfico (figura 4) ilustra la participación en forma más general, incluyendo el uso de las redes digitales. En este caso se clasifica la participación de los noveles investigados en "baja”, "media", "alta” y "sin participación”. En el gráfico puede observarse que la participación se divide en una mayoría ubicados en lo que se calificó como participación alta, dos subgrupos vacíos (media y sin participación) y un docente con participación baja.

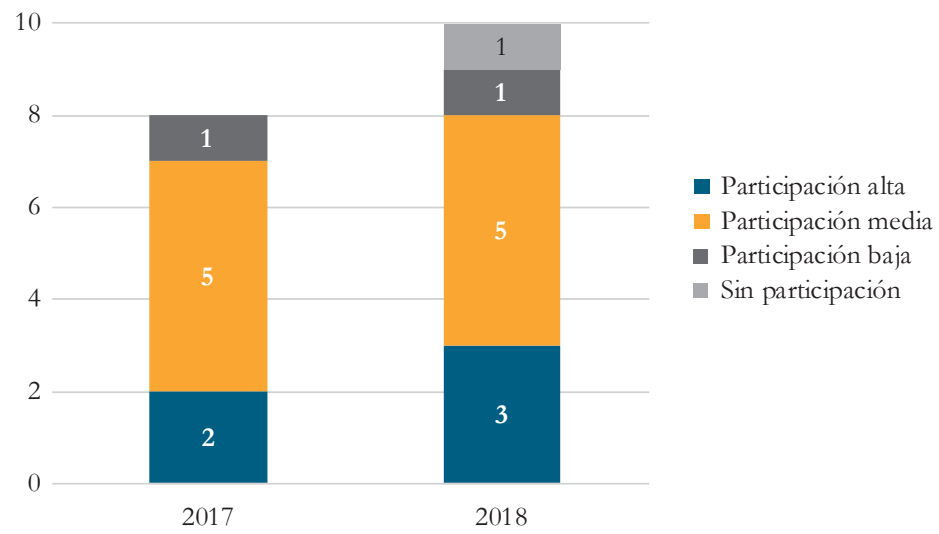

Figura 4: Participación de los docentes noveles en el programa de acompañamiento.. Elaboración propia en base a datos obtenidos en la investigación

Tres (3) de los cuatro noveles a quienes se les hizo seguimiento fueron clasificados en el grupo de participación alta, dado que asistieron a al menos al $50 \%$ de los encuentros presenciales, con dos (2) de ellos a más del $75 \%$ de los realizados. Esos mismos tres docentes tuvieron alta participación en las redes: grupo en CREA y Whatsapp. Hacia el final del período de acompañamiento uno de ellos gestionó y dictó un taller, y el tercero gestionó un último encuentro, no previsto por las investigadoras, frente a un emergente. El cuarto docente, dejó paulatinamente de participar en la red, al menos en forma significativa (participó en forma activa en el grupo de Whatsapp pero con contenidos de escasa trascendencia). 
Finalmente se consideró de interés incluir un gráfico con la participación de todos los docentes noveles convocados en el programa de acompañamiento, discriminado por año. Esta información se muestra en la figura 5, en forma similar al anterior pero para la población objetivo en su totalidad (ocho docentes en 2017 y diez en 2018). En este caso puede observarse que la participación se divide en una gran mayoría ubicados en lo que se calificó como participación media, y dos subgrupos más pequeños con alta y baja participación. Un docente no participó de ninguna instancia de acompañamiento en 2018. La diferencia numérica de la población objetivo se debe al alta de tres nuevos egresados y a la baja por traslado a otro departamento de un integrante de 2017.

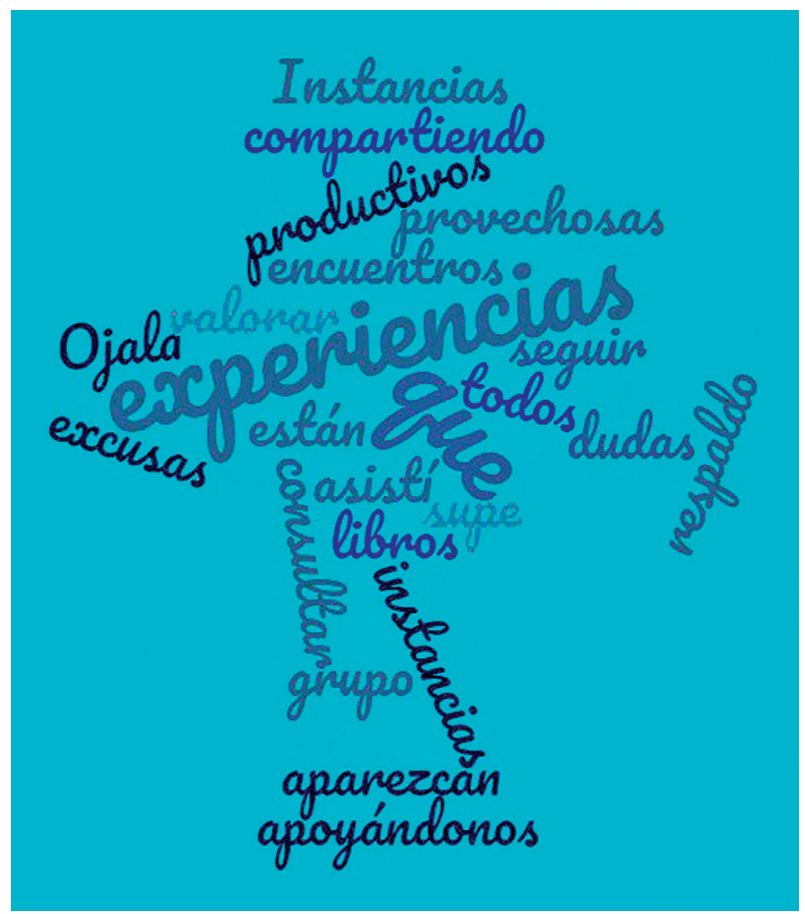

Figura 5: Participación global del grupo completo de docentes noveles en 2017 y en 2018. Elaboración propia en base a datos obtenidos

Del análisis de la información presentada más lo recabado en visitas y entrevistas se desea destacar los siguientes puntos:

- En general puede decirse que participación de los noveles en el programa fue discontinua y variada. Existieron momentos de gran participación y entusiasmo y otros donde se hacía difícil organizar actividades. En cuanto a las diferencias entre participantes, los noveles más participativos se mantuvieron interesados los dos años analizados, y lo hicieron en la mayoría de los ámbitos: encuentros, participación en redes virtuales, gestión de dispositivos de acompañamiento. Es posible que el hecho de ser seleccionados para el seguimiento haya influido la mayor participación de este subgrupo frente al colectivo de noveles.

- Los encuentros presenciales contribuyeron a fortalecer el vínculo en el grupo y fueron las instancias más ricas de intercambio y apoyo mutuo. Esta información surge de la observación participante de las investigadoras y de las encuestas finales realizadas a los docentes investigados.

- Por un proceso similar se constata que la plataforma CREA no se impuso como andamiaje motivador para compartir experiencias. El uso de la misma fue a ins- 
tancias del equipo de investigadoras y funcionó como repositorio de materiales y recursos. Si bien fue gestionada desde el inicio por un novel docente, y permitió organizar la información compartida, no logró convertirse en un espacio de discusión o reflexión sobre la práctica. En cambio, el grupo de WhatsApp tuvo mayor protagonismo y dinamismo. Contribuyó fuertemente en aspectos vinculares y, a pesar de ser un medio efímero, la mayor parte de la información compartida pasó por este medio. De hecho, el grupo permanece activo en la actualidad, a casi dos años de finalizada la investigación.

- A partir de las visitas realizadas, y de las encuestas y entrevistas surge que los docentes noveles asisten poco o nada a los laboratorios. Declaran estar más cómodos trabajando en el salón de clase, dado que se sienten interpelados en los laboratorios y no reciben el apoyo esperado. Ello trae aparejado dos consecuencias: un menor énfasis en la actividad experimental y una menor interacción con los docentes y preparadores de la institución. Esta percepción fue en general compartida por los otros actores del sistema educativo entrevistados. Sin embargo, también queda sobre la mesa que no es solo una característica de los docentes noveles.

- En una relación dialéctica causa-efecto respecto al punto anterior se observó que gran parte de los noveles del grupo de acompañamiento presentaron dificultades para posicionarse como docente-par frente al colectivo docente. La contracara de esta observación se manifestó en varias entrevistas a preparadores y colegas, donde surge en el discurso del valor de la experiencia. La "ayuda" a los noveles pasaría, de acuerdo a los expertos, más bien por compartir los conocimientos adquiridos por la práctica en la medida que los noveles "se acerquen y pidan ayuda" y no tanto en un apoyo para la innovación. Se parte de la base que "no saben" utilizar el instrumental de los laboratorios por ejemplo, lo que concuerda y confirma la visión del relacionamiento desde el novel.

- A lo largo del programa se pudo constatar el desarrollo de autonomía para gestionar encuentros tanto de formación como de reflexión. En las entrevistas y encuestas finales la mayor parte del grupo estudiado se muestra encaminado hacia una autonomía de grupo en el trabajo en redes y hacia un compromiso en su formación permanente y en la reflexión continua sobre sus prácticas. Sin embargo, las redes docentes noveles - expertos no fue el campo preferido. En general prefieren trabajar en redes propias, sin docentes expertos, y consultar unilateralmente al experto cuando se le presentan dudas o requieren apoyo.

La siguiente nube de palabras (figura 6) muestra el resultado de las expresiones de los noveles docentes al ser consultados sobre el acompañamiento en su etapa final.

\section{Reflexiones finales}

A lo largo del desarrollo del programa de acompañamiento se constataron continuamente dificultades para concretar las instancias presenciales formativas: ateneos, talleres y otros. En algunos casos las reuniones coincidían con reuniones de centro de diferentes instituciones y el margen de horario disponible era muy corto para la actividad a realizar. Por lo tanto sería conveniente que se gestionan espacios ins- 
titucionales con fines de acompañamiento a docentes principiantes para que estos programas sean viables, eficientes y se mantengan activos a lo largo del tiempo.

En este programa no se logró el involucramiento significativo de otros docentes expertos, a pesar de haber sido invitados a participar en diferentes instancias su asistencia a los encuentros fue esporádica. Sin embargo, el grupo de investigación considera que el involucramiento de los docentes de las instituciones en las cuales los noveles se desempeñan es imprescindible para poder acompañar sus primeros pasos en la institución y que deberá re-pensarse los medios y dinámicas para integrarlos. De esta forma, se afianzarían los vínculos personales y profesionales, que habilitarán el apoyo necesario y el intercambio entre pares dando cuerpo a una formación continua y a la revisión crítica de las prácticas entre todos. Otro aspecto significativo es la conveniencia de establecer canales de comunicación efectivas entre las diferentes Instituciones Educativas donde se desempeñan los docentes, ya sean dependientes del CES (Consejo de Educación Secundaria) o del CFTP (Consejo de Formación Técnico Profesional), con los docentes de Didáctica y estudiantes que se encuentren cursando su último año de práctica pre profesional. Este aspecto también puede contribuir a la inserción de los noveles en dichas instituciones, forjando alianzas para la instrumentación de diversos programas de acompañamiento.

Finalmente, a pesar de las dificultades ya expresadas, en el trascurso de la investigación se pudo constatar que las redes interpersonales permitieron una reflexión conjunta sobre las prácticas, fortaleciendo los vínculos entre docentes y habilitando posibles mejoras en la enseñanza de la física. En la búsqueda de mantener activas estas redes se ve como fundamental comprometer a las autoridades y al colectivo docente en su conjunto.

\section{Bibliografía}

Barbier, J. M. (1999): Prácticas de formación: evaluación y análisis. Buenos Aires. Davini, M. (2015): La Formación en la práctica docente. Buenos Aires. Paidós

De Lella, C. (1999): Modelos y tendencias de la Formación Docente en I Seminario Taller sobre Perfil del Docente y Estrategias de Formación. Recuperado de http://www.oei.es/historico/cayetano.htm

Klein, G. (2012): Didáctica de la Física. Recuperado de http://www.anep.edu.uy/ ipa-fisica/document/material/cuarto/2008/didac 3/did fis.pdf

Maciel de Oliveira, C. (2016). Diseño y preevaluación de un programa de desarrollo profesional para profesores principiantes de educación secundaria. Tesis doctoral. UNED.

Nossar, K. (2016): Mano a mano con los noveles: límites a la intervención y potencialidad del programa de acompañamiento. En: Nossar, K. y Sallé, M. Docentes noveles: investigaciones, experiencias e innovaciones (pp. 101-110). Montevideo, CFE- ANEP.

Nossar, K. y Sallé, C. (2017). La potencialidad de las interacciones entre educadores expertos y noveles Aportes teóricos, dispositivos de trabajo y producciones. Recuperado de https://oei.org.uy/uploads/files/programs/1/projects/13/ publicacion-proyecto-noveles.pdf

Souto, M. (1999). Un dispositivo de formación el seminario-taller Grupos de Formación. Buenos Aires. 
Vera-Godoy,R.(2008):Eltallerdeeducadores, unaherramientaconceptualymetodológica para el análisis de la propia práctica. Buenos Aires. Recuperado de https:// cedoc.infd.edu.ar/upload/Introduccion20al20taller20de20educadores1.pdf 